\title{
1608+656: A GRAVITATIONALLY LENSED POSTSTARBURST RADIO GALAXY
}

\author{
C. D. Fassnacht, ${ }^{1}$ D. S. Womble, ${ }^{1}$ G. Neugebauer, ${ }^{1}$ I. W. A. Browne, ${ }^{2}$ \\ A. C. S. Readhead, ${ }^{1}$ K. Matthews, ${ }^{1}$ \\ AND T. J. PEARSON ${ }^{1}$ \\ Received 1995 December 5; accepted 1996 January 17
}

\begin{abstract}
The gravitational lens system $1608+656$ displays four flat-spectrum, pointlike components that are the images of the unresolved core of a double-lobed radio source. The lensing mass is a galaxy at $z=0.630$. New spectra of this system enable us to determine a conclusive redshift of 1.394 for the lensed object. The spectra show prominent high-order Balmer absorption lines and $\mathrm{Mg}$ II absorption. These lines, and the absence of [O II] emission, indicate that this is a poststarburst or $\mathrm{E}+\mathrm{A}$ galaxy. It is unique among lensed objects in not being a quasar and among $\mathrm{E}+\mathrm{A}$ galaxies in having the highest known redshift. Even allowing for lens magnification, the lensed object is a very luminous galaxy, with an absolute magnitude, $M(r)=-22.8$ mag. The deconvolved infrared image indicates that the galaxy may be slightly resolved. The radio luminosity density of the lobes is $L_{1.4}=5.78 \times 10^{25} \mathrm{~W} \mathrm{~Hz}^{-1}$, which puts the source on the boundary between FR I and FR II radio galaxies. Together with the redshift for the lens and a satisfactory mass model, the determination of the lensed object redshift makes this system an excellent candidate for measuring $H_{0}$.
\end{abstract}

Subject heading: gravitational lensing

\section{INTRODUCTION}

The quadruply-imaged gravitational lens $1608+656$ was discovered as part of the Cosmic Lens All Sky Survey (CLASS; Myers et al. 1996), a large-scale search for gravitational lenses among flat-spectrum radio sources using the VLA. ${ }^{3}$ CLASS was designed to find systems with the lensing potential dominated by a single galaxy. The expected yield from the survey is 10-15 new lenses. Because single-galaxy lensing potentials are fairly straightforward to model, CLASS provides a powerful method for determining galaxy masses and mass-to-light ratios. The $8.4 \mathrm{GHz}$ radio map of $1608+656$ shows four flatspectrum, pointlike components (Myers et al. 1995, hereafter Paper I), while $1.4 \mathrm{GHz}$ maps show that the source being lensed is the core of a classical double radio source (Snellen et al. 1995). Optical and infrared images (Paper I) show the same morphology as the $8.4 \mathrm{GHz}$ radio map and additionally show the lensing galaxy. Spectra of this object gave a redshift $z=0.630$ for the lensing galaxy (Paper I), but did not yield a conclusive redshift for the background source. A simple mass model derived in Paper I reproduces the location of the images and the lensing galaxy. Thus, this is an excellent system for the determination of the Hubble constant $H_{0}$, provided that a redshift for the lensed object and a time delay can be measured. This paper reports on spectroscopic observations made with the Palomar $5 \mathrm{~m}$ Telescope that provide a redshift for the lensed object. Optical and infrared imaging are also reported. We have assumed $H_{0}=100 h \mathrm{~km} \mathrm{~s}^{-1} \mathrm{Mpc}^{-1}$ and $q_{0}=0.5$ throughout this Letter.

\footnotetext{
${ }^{1}$ Palomar Observatory, California Institute of Technology, 105-24, Pasadena, CA 91125.

2 NRAL Jodrell Bank, University of Manchester, Macclesfield, Cheshire SK11 9DL, UK.

${ }^{3}$ The VLA is a facility of the National Radio Astronomy Observatory, which is operated by Associated Universities, Inc., under contract with the National Science Foundation.
}

\section{OBSERVATIONS AND DATA REDUCTION}

\subsection{Spectroscopy}

Optical spectra of $1608+656$ were obtained on 1995 July 21 and 23 with the double spectrograph on the Palomar $5 \mathrm{~m}$ Telescope. A total of five $2000 \mathrm{~s}$ observations were made, covering the wavelength range 3400-9700 $\AA$. The observing conditions were excellent, with $\sim 0$ ". 8 FWHM seeing and no visible clouds. In order to minimize the contribution of light from the foreground lensing galaxy, the slit was aligned at a position angle of $160^{\circ}$ centered on lens components $\mathrm{A}$ and B (see Fig. $2 a$ below). A slit width of 1."0 was used together with a 300 lines $\mathrm{mm}^{-1}$ grating in the blue and a 158 lines $\mathrm{mm}^{-1}$ grating in the red to produce a spectral resolution of $5.4 \AA$ FWHM for $\lambda<5100 \AA$ and $15.2 \AA$ FWHM for $\lambda>5100 \AA$.

The resulting data were reduced using the VISTA and IRAF software packages. Bias subtraction and flat-fielding were done using standard procedures. Because of the faintness of the object relative to the sky background, the spatial curvature of the spectrum was traced and removed using a standard star profile. The two-dimensional spectra were cleaned of cosmic rays and extracted to one-dimensional using an optimal weighting algorithm similar to that described by Horne (1986); spectra of the background sky and $1 \sigma$ uncertainties based on Poisson statistics were also extracted by this routine. Flexure corrections to the wavelength scales were applied using prominent night-sky lines, and the data were flux calibrated using standard star exposures taken during the evening and morning twilights. Atmospheric absorption bands were removed from the individual exposures using template spectra that were produced by scaling the appropriate portions of standard star spectra to the effective object air masses. All of the resulting spectra for $1608+656$ were weighted according to their signal-to-noise ratio and added; a portion of the combined spectrum is shown in Figure 1. No significant features are evident at wavelengths other than those shown in this figure. 


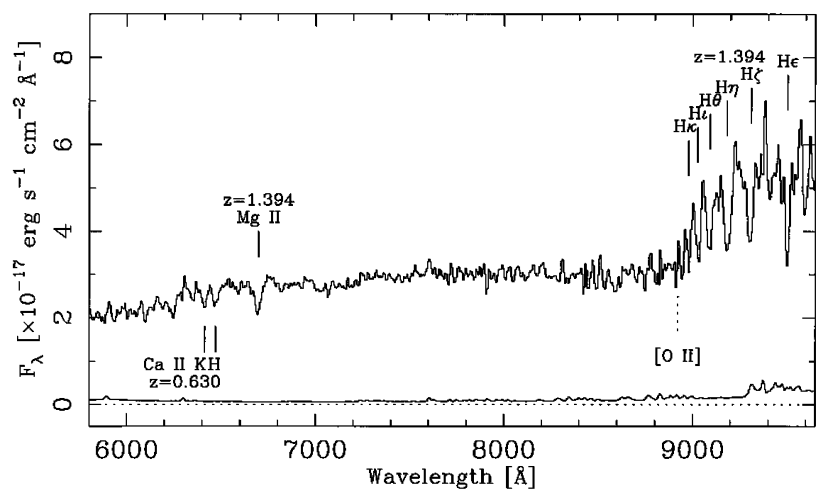

FIG. 1.-Optical spectrum of the gravitational lens $1608+656$ with the slit positioned to maximize the background source light and minimize the lensing galaxy light. The wavelengths are as observed in air; features belonging to the background source, $z=1.394$, and the lensing galaxy, $z=0.630$, are labeled. The solid vertical lines mark the positions of detected absorption features at both redshifts whereas the dotted line marks the expected position of [O II] $\lambda 3726.6$ emission at $z=1.394$. The lower spectrum designates the $1 \sigma$ uncertainties associated with the object spectrum.

\subsection{Infrared Imaging}

An image of $1608+656$ was taken on the night of 1995 July 18 with the Cassegrain infrared camera at f70 focus of the Palomar $5 \mathrm{~m}$ Telescope. The camera scale was 0 ". $125 \mathrm{pixel}^{-1}$ and the median seeing was 0.7 FWHM in the $K$-short $(K \mathrm{~s})$ filter band which admits radiation between 2.00 and $2.30 \mu \mathrm{m}$. Twenty-five exposures, each of $40 \mathrm{~s}$, were obtained using an offset guider which applied "tip-tilt" corrections based on the visual position of the guide star. Three similar exposures of a star $4^{\prime}$ from the object were interleaved with the 25 object observations to provide a point spread function (PSF); the guide star used for $1608+656$ was also used for the PSF observations. The images were linearized, flat-fielded, and sky-subtracted following standard procedures. The 25 images were sorted in order of sharpness, and the eight sharpest images were combined in the mosaic image shown in Figure $2 a$ (Plate L14). Flux calibration was not done because the conditions were not photometric.

The PSF was normalized and convolved with a model containing the positions and intensities of the four unresolved radio components. The intensities were taken from a VLA image of $1608+656$ that was observed on 1995 July 6. The resulting image was scaled to the level of the $K$ s image and subtracted from it, giving the residual image shown in Figure $2 b$ (Plate L15). A Lucy-Richardson deconvolution (Lucy 1974; Richardson 1972; Snyder, Hammond, \& White 1993) on the $K$ s image was also carried out using the normalized PSF. The result from 40 iterations is shown in Figure $2 c$ (Plate L16).

\subsection{Optical Imaging}

The $1608+656$ field was observed with the imaging CCD camera on the Palomar $1.5 \mathrm{~m}$ Telescope on 1995 April 24-27, using Gunn $r$ and $i$ filters. Three $800 \mathrm{~s}$ and two $600 \mathrm{~s}$ exposures were taken of $1608+656$. The limiting magnitudes for detecting unresolved objects were $m_{r} \sim 22.2$ and $m_{i} \sim 21.9$. A standard star was observed before and after the $1608+656$ exposures. The camera scale was 0 ".372 pixel $^{-1}$, giving a field of view of $13^{\prime} \times 13^{\prime}$. The first three nights were photometric, with average seeing of 1."4 FWHM, which allowed a determination of colors for the object. The images were bias-subtracted and flat-fielded using IRAF. In a similar manner to that described above, residual images were created by subtracting a model of the four source components from the $r$ and $i$ images taken in the best seeing. The lens galaxy flux density was derived from the residual image and the source flux density from the model fit. Magnitudes were derived using the "apphot" package in IRAF and are given in the following section.

\section{RESULTS AND DISCUSSION}

In the final spectrum, the blended $\mathrm{Mg}$ II doublet $\lambda \lambda 2795.5$, 2802.7 is identified in absorption at $6696.0 \AA$, as are the Balmer absorption lines $\mathrm{H} \epsilon \lambda 3970.1, \mathrm{H} \zeta \lambda 3889.1, \mathrm{H} \eta \lambda 3835.4$, $\mathrm{H} \theta \lambda 3797.9, \mathrm{H} \iota \lambda 3770.6, \mathrm{H} \kappa \lambda 3750.2$ at $\lambda=9500.4,9303.3$, $9182.3,9088.4,9030.8$, and $8979.6 \AA$. These lines yield an unambiguous redshift for the background source of $z=1.394$. No [O II] $\lambda 3726.6$ emission is detected at this redshift to a rest equivalent width limit of $1 \AA(3 \sigma)$. For comparison, the $\mathrm{H} \zeta$ line has a rest equivalent width of $3.9 \pm 0.5 \AA$. A Ca II K and $\mathrm{H} \lambda \lambda 3933.7,3968.5$ doublet is also clearly seen at $\lambda=6411.8$, $6469.4 \AA$; this was seen in Paper I and is associated with the lensing galaxy at a redshift of $z=0.630$. It should be noted that the $z=1.394 \mathrm{Mg}$ II absorption occurs at the same wavelength as the $\mathrm{H} \delta$ absorption from the $z=0.630$ lensing galaxy. This causes the spectrum of the lensing galaxy (see Paper I) to have deceptively strong $\mathrm{H} \delta$ absorption. However, the $z=0.630$ [O II] emission and $\mathrm{Mg}$ II absorption seen in the lens spectrum (Paper I) are not associated with any lines from the background source.

The lensed object is not what one would expect for the host of a relatively powerful radio source. Instead of strong emission lines, the spectrum is more like that of an A star, characteristic of an " $E+A$ " or "poststarburst" galaxy (Dressler \& Gunn 1983; Oegerle et al. 1991). These galaxies are composed of old stars typical of elliptical galaxies ("E") and stars thought to have formed in a burst within the past $10^{9}$ yr ("+ A"). Dressler \& Gunn (1983) proposed that the bursts of star formation in $\mathrm{E}+\mathrm{A}$ galaxies may have been triggered by ram pressure compression of gas in clusters or through galaxy-galaxy interactions and/or mergers. Because mergers and interactions may be a cause for the onset of radio emission in galaxy nuclei, it is possible that the same mechanism that produced this $\mathrm{E}+\mathrm{A}$ galaxy is also responsible for origin of the nuclear radio source. We note that the redshift of $z=1.394$ for $1608+656$ is higher than that of CL $1601+4253$ No. 105 , which at $z=0.7367$ was previously the $\mathrm{E}+\mathrm{A}$ galaxy with the highest known redshift (Dressler \& Gunn 1992).

We know of no other radio galaxy that has such a spectrum, although we are aware that the host around the quasar 3C 48 (Boroson \& Oke 1982) has Balmer absorption lines typical of A stars. What makes $1608+656$ so unusual for an active galaxy is the absence of narrow emission lines. If it were like $3 \mathrm{C} \mathrm{48,}$ its nucleus must be exceedingly well hidden. The unusual spectrum might be a result of selective magnification in the lensing of one part of the host galaxy image at the expense of the nucleus. However, we think this unlikely as the similarity of the radio and optical flux density ratios implies that the emission at both wavelengths comes from the same region; i.e., the nucleus of the galaxy.

With the source redshift determined, it is possible to calculate the radio luminosity and linear size of the source. Snellen et al. (1995) found that the total $1.4 \mathrm{GHz}$ flux density from the radio lobes was $12 \mathrm{mJy}$ and that the peaks in the lobes 
PLATE L14

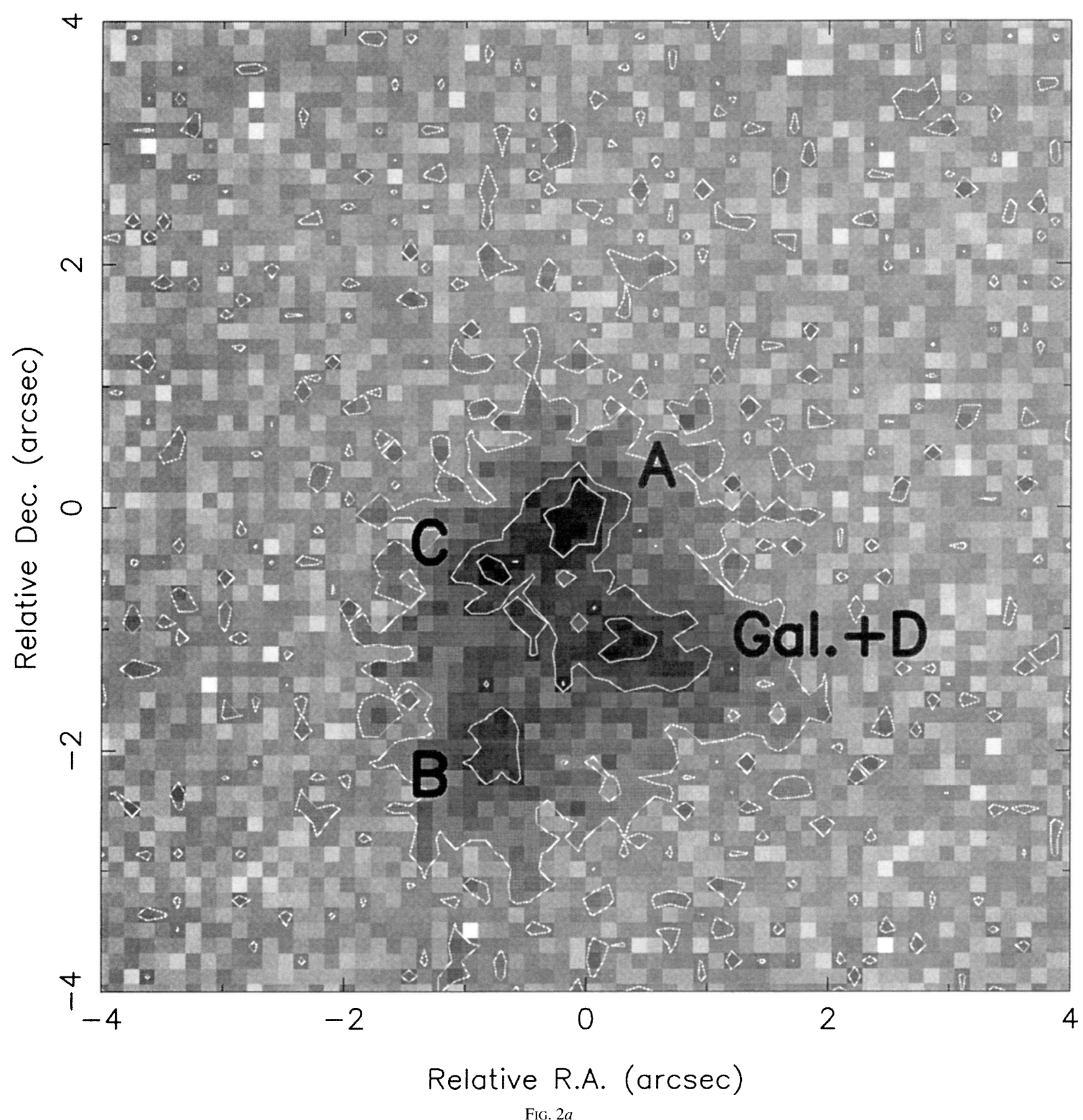

Fig. 2.- (a) Infrared $K$ s-band image of the quadruple lens $1608+656$. North is up, and east is to the left. (b) Residual image of $1608+656$ produced by the subtraction of a scaled radio model from the field. Resulting image is that of the lensing galaxy. (c) Lucy-Richardson deconvolution of $K$ s-band image.

FASSNACHT et al. (see 460, L104) 
PLATE L15

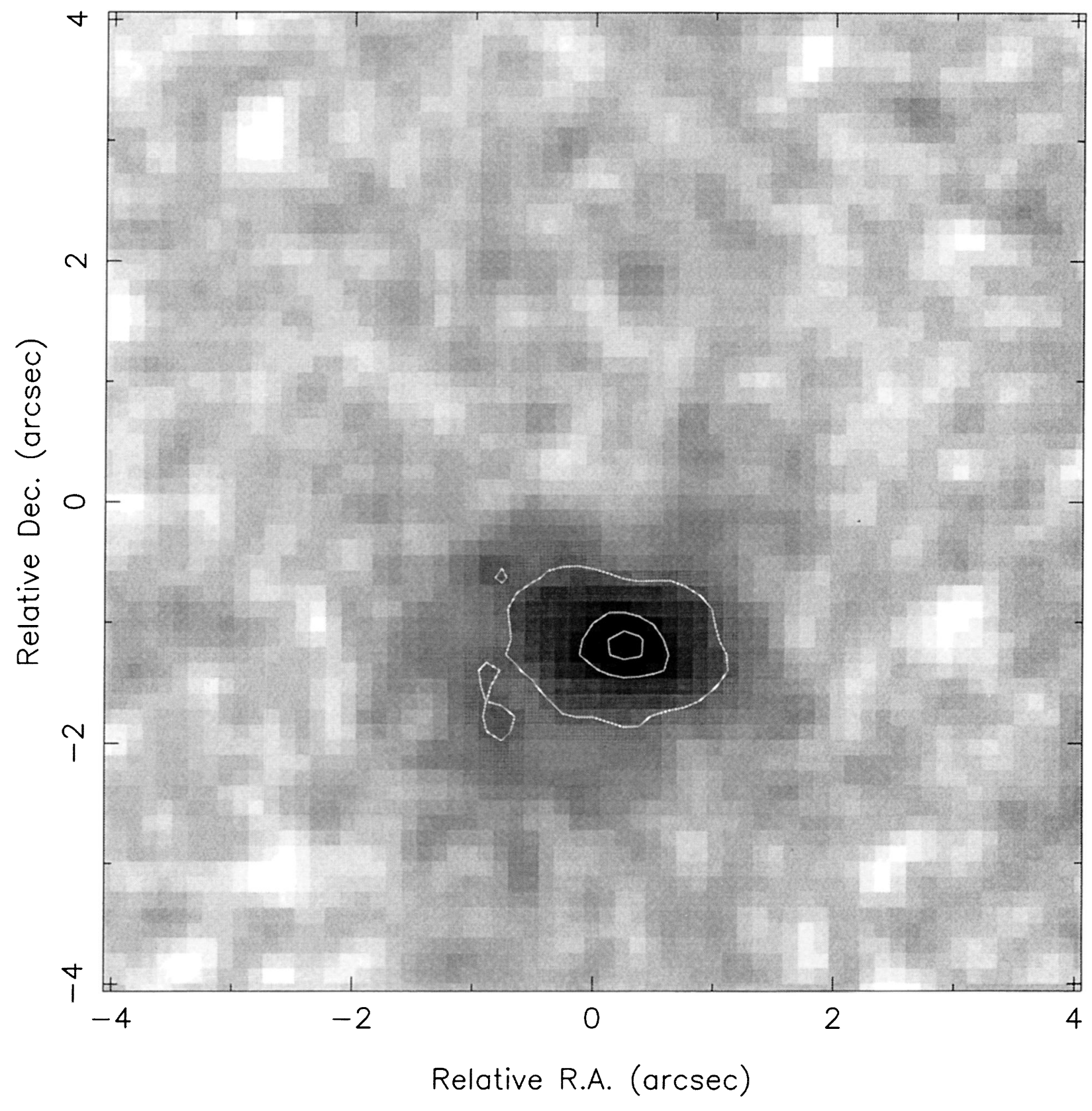

FIG. $2 b$

FASSNACHT et al. (see 460, L104) 


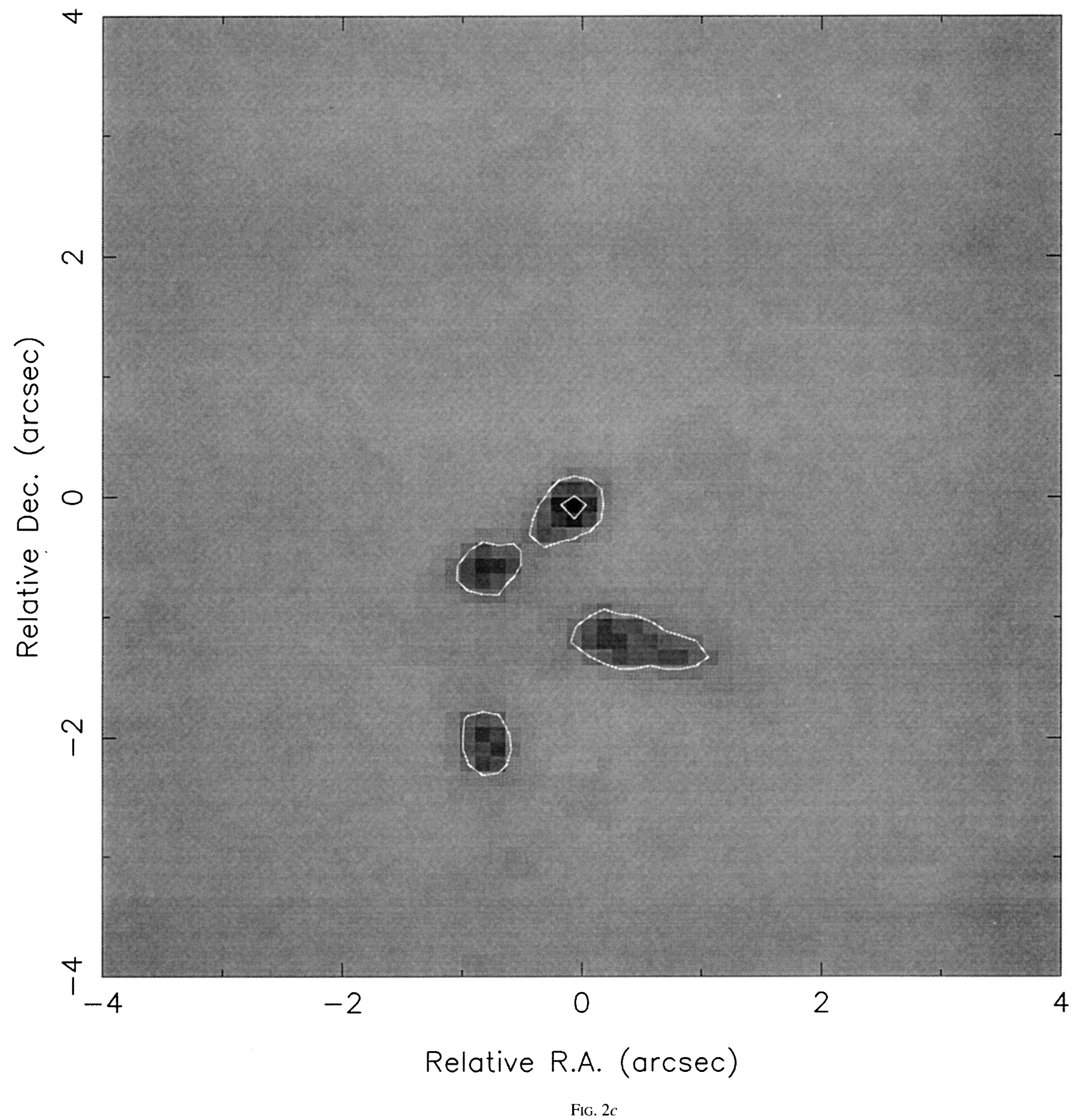

FASSNACHT et al. (see 460, L104) 
were separated by $50^{\prime \prime}$. Using the redshift $z=1.394$ for the source and assuming a spectral index $\alpha=-0.5$ (where $S_{\nu} \propto \nu^{\alpha}$ ) gives an emitted luminosity density at $1.4 \mathrm{GHz}$ of $L_{1.4}=5.8 \times 10^{25} \mathrm{~W} \mathrm{~Hz}^{-1}$ and a projected peak separation of $215 h^{-1} \mathrm{kpc}$. The calculated luminosity density puts $1608+656$ on the boundary between FR I and FR II radio galaxies (Fanaroff \& Riley 1974), which is usually taken as $10^{25} \mathrm{~W} \mathrm{~Hz}^{-1}$ at $1.4 \mathrm{GHz}$.

Optical magnitudes were derived from the residual images (for the lens) and the scaled radio models (for the background source), as discussed in the previous section. The lens magnitudes are $m(r)=20.0 \mathrm{mag}, r-i=0.7 \mathrm{mag}$ and the total source magnitudes, from combining all four components, are $m(r)=20.2 \mathrm{mag}, r-i=0.6 \mathrm{mag}$. To obtain luminosities we have calculated $k$-corrections for the $r$ and $i$ bands using a composite stellar spectrum of type A9 to F0 V taken from Silva \& Cornell (1992) for 1100-3200 ̊ and from Heck et al. (1984) for 3750-8900 $\AA$. This spectrum closely matches that of the source in the vicinity of the Balmer lines; it is a much better representation of the spectral energy distribution than any of the galaxy SEDs given by Coleman, Wu, \& Weedman (1980). The resulting $k$-corrections for $z=1.394$ are $k(r)=1.4$, $k(i)=0.6 \mathrm{mag}$. In order to calculate the intrinsic luminosity of the source, we must also account for the magnification due to lensing. From the lens model given in Paper I, the total magnification is a factor of 5.9 , or 1.9 mag. Provided that this magnification limit holds for $1608+656$, the implied absolute magnitude for the source is $M(r)=-22.8-5 \log h^{-1}$. The source is considerably brighter than a typical $L^{*}$ galaxy, where $M(r)^{*}=-20.2-5 \log h^{-1}$ (Loveday et al. 1992; Frei \& Gunn 1994), and it approaches the luminosities of the brightest known galaxies. The magnification is calculated assuming that the background source is pointlike. If the source is extended, as may be the case for a galaxy, this value for the magnification is only valid when $r \mu \ll D$, where $r$ is the intrinsic source size, $\mu$ is the magnification, and $D$ is the distance between images. For the most magnified component $\mathrm{A}$ and its nearest neighbor $\mathrm{C}$, this limit corresponds to an intrinsic source size, $r \ll 0$ ". 34, or a projected distance of $r \ll 1.5 h^{-1} \mathrm{kpc}$ at $z=1.394$. If the intrinsic source size is larger than this, then the total magnification will be smaller than our model predicts. The result is that the intrinsic source luminosity could be greater than that derived above. However, the clean residuals displayed in Figure $2 b$ demonstrate that the magnifications derived from the radio data are approximately correct for modeling the infrared image. Thus we conclude that the calculated source luminosity is fairly accurate. However, strictly speaking, it is a lower limit.

Although we identify $1608+656$ as an E + A galaxy based on the strong Balmer absorption lines, there is little spectral evidence for the presence of an old stellar population. The majority of features associated with older stars have wavelengths longward of our spectral coverage. The wavelengths of the $\mathrm{Ca}$ II $\mathrm{K}$ and $\mathrm{H}$ doublet at $z=1.394$ are covered by the spectrum, but if the doublet is present it is very weak and may be contaminated by atmospheric absorption. The lack of strong evidence for older stars raises the possibility that $1608+656$ has had only one burst of star formation. One check on this possibility is to compare the colors of $1608+656$ with those of young star clusters to see if a system composed solely of young stars could reproduce the very red $i-K$ color that is seen in $1608+656$. We estimated the integrated flux in bands corresponding to $K$ and $i$ for $z=1.394$ of seven young clusters
(Bica, Alloin, \& Schmitt 1994; Bica \& Alloin 1986, 1987). These gave $i-K$ colors ranging from -0.9 mag for the youngest cluster $\left(8 \times 10^{6} \mathrm{yr}\right)$ to $0.2 \mathrm{mag}$ for the oldest clusters $\left(1-2 \times 10^{9} \mathrm{yr}\right)$. In comparison, $1608+656$ has $i-K=2.7 \mathrm{mag}$, where the $\mathrm{K}$ magnitude is from Paper I. Thus the colors of $1608+656$ are significantly redder than expected for only a single population of young stars. This implies that an old stellar population is also present in $1608+656$ unless the continuum is reddened by about $\mathrm{E}(i-K)=2.5$ as the light passes through the lens. We think that this is unlikely because such reddening would imply an extinction of $A_{r}=4.0$, assuming a "van de Hulst No. 15" theoretical extinction curve (van de Hulst 1949). This gives a corrected absolute magnitude for the background source of $M(r)=-26.8-5 \log h^{-1}$, which is unrealistically bright.

The infrared image of $1608+656$ shows that the lensing galaxy is clearly separated from the three brightest components of the multiply lensed background source (A, B, and C; see Fig. 2a) and that the three components are also well separated from each other. The deconvolved image (Fig. $2 c$ ) matches the structure in the radio map (see Paper I) extremely well. This is what is expected if the majority of the infrared light originates from the same region as the radio emission. There is a hint, however, of some extended emission between components $\mathrm{A}$ and $\mathrm{C}$, and component $\mathrm{B}$ appears to be somewhat elongated in the north-south direction. Another possible indication that the background source is extended is that the residual image (Fig. $2 b$ ) shows an excess near the location of component $\mathrm{B}$ in addition to the lensing galaxy. It is possible that we are resolving more than just the nuclear region of the background source. This is consistent with the "stellar" nature of the spectrum of the lensed source.

In Paper I, the mass model for the lensing galaxy was derived from the positions and flux densities of the components in the original radio image. The lens can be modeled as a single galaxy with a central mass density that can be expressed in a redshift-independent form as

$$
\rho_{0}=\frac{9 \sigma^{* 2}}{4 \pi G r_{c}^{2}} d_{\mathrm{ls}}, \quad d_{\mathrm{ls}}=\frac{D_{\mathrm{s}}}{D_{\mathrm{ls}}},
$$

where $D_{\mathrm{s}}$ and $D_{\mathrm{ls}}$ are the observer-source and lens-source angular diameter distances, $G$ is the gravitational constant, $\sigma^{*}$ is related to the central velocity dispersion by $\sigma=\sigma^{*}\left(d_{\mathrm{ls}}\right)^{1 / 2}$, and $r_{c}$ is the scale length of the mass distribution. The redshifts of $z=0.630$ for the lensing galaxy and $z=1.394$ for the source give $D_{1}=798 h^{-1} \mathrm{Mpc}, D_{\mathrm{s}}=886 h^{-1} \mathrm{Mpc}$, and $D_{\mathrm{ls}}=343 h^{-1}$ Mpc. This gives a central mass density of $\rho_{0}=1.25 \times 10^{10} h^{2}$ $M_{\odot} \mathrm{kpc}^{-3}$ and a mass within the Einstein ring radius $\left(\theta_{\mathrm{E}}=1\right.$ ". 1$)$ of $3.05 \times 10^{11} h^{-1} M_{\odot}$. Using the $K$-band luminosity of $7.7 \times 10^{8} L_{\odot}$ within $\theta_{\mathrm{E}}$ from Paper I gives a mass-to-light ratio within the Einstein radius of $(M / L)_{\mathrm{E}}=6.98 h M_{\odot} / L_{\odot}$. The expected time delays are $t_{\mathrm{C}}-t_{\mathrm{A}}=4.0 h^{-1}$ days and $t_{\mathrm{A}}-t_{\mathrm{B}}=40.0 h^{-1}$ days.

The large derived mass for $1608+656$ is comparable to the largest cluster ellipticals and could indicate that a pair of galaxies or a merging galaxy system is doing the lensing. However, the $K$ s band image of $1608+656$ does not support this suggestion, and the single galaxy model derived in Paper I is consistent with all the present data. In addition, there is no indication of a galaxy cluster in the $r$ and $i$ band images. Hence, any further refinement to the model will have to await new information. 


\section{CONCLUSIONS}

Our observations of the lensed system $1608+656$ have given a redshift of $z=1.394$ for the background source. The background source is unique among lensed objects in that it is a poststarburst radio galaxy and not a quasar. The new observations are consistent with the mass model derived in Paper I. Hence, three of the four pieces of information needed to determine $H_{0}$ from the system are now available: the redshift of the lens, the redshift of the background source, and a working mass model; what is missing is the measurement of a time delay. A monitoring program is underway, and there is a strong possibility for measuring a time delay and finding $H_{0}$ from this system.

For valuable discussions, we thank Roger Blandford, David Hogg, Steve Myers, Ann Zabludoff, Neal Jackson, and Lee Armus. We are grateful to Greg Taylor for helping with the optical observations. We thank the staff at Palomar Observatory for their assistance during our observing runs. CLASS and Infrared astrophysics at Caltech are supported by grants from the NSF. C. D. F. is supported by a NSF Graduate Research Fellowship. D. S. W. acknowledges support of a Hubble Fellowship provided by NASA through grant HF-1040.01-92A from the STScI, which is operated by AURA.
Bica, E., \& Alloin, D. 1986, A\&A, 162, 21

.1987, A\&A, 186, 49

Bica, E., Alloin, D., \& Schmitt, H. R. 1994, A\&A, 283, 805

Boroson, T. A., \& Oke, J. B. 1982, Nature, 296, 397

Coleman, G. D., Wu, C.-C., \& Weedman, D. W. 1980, ApJS, 43, 393

Dressler, A., \& Gunn, J. 1983, ApJ, 270, 7

Dressler, A., \& Gunn, J. E. 1992, ApJS, 78, 1

Fanaroff, B. L., \& Riley, J. M. 1974, MNRAS, 167, 31

Frei, Z., \& Gunn, J. E. 1994, AJ, 108, 1476

Heck, A., Egret, D., Jaschek, M., \& Jaschek, C. 1984, A\&AS, 57, 213

Horne, K. 1986, PASP, 98, 609

Loveday, J., Peterson, B. A., Efstathiou, G., \& Maddox, S. J. 1992, ApJ, 390, 338

\section{REFERENCES}

Lucy, L. B. 1974, AJ, 79, 745 Myers, S. T., et al. 1995, ApJ, 447, L5 (Paper I)

Oegerle, W. R., Hill, J. M., \& Hoessel, J. G. 1991, ApJ, 381, L9

Richardson, W. H. 1972, J. Opt. Soc. Am., 62, 55

Silva, D. R., \& Cornell, M. E. 1992, ApJS, 81, 865

Snellen, I. A. G., de Bruyn, A. G., Schilizzi, R. T., Miley, G. K., \& Myers, S. T. 1995, ApJ, 447, L9

Snyder, D. L., Hammoud, A. M., \& White, R. L. 1993, J. Opt. Soc. Am. A, 10, 1014

van de Hulst, H. C. 1949, Rech. Astr. Obs. Utrecht, 11, Part 1, 1 